\title{
Image Processing with the Specific Focus on Early Tumor Detection
}

\author{
Mashal Tariq, Attaullah Khawajah, and Munawer Hussain
}

\begin{abstract}
The primary purpose of this paper is to elaborate upon and to take a step ahead on the research done in the field of Image Processing with a focus on Early Tumor Detection through the use of Magnetic Resonance Imaging (MRI) and Image Processing Tool Box of MATLAB. The technique which the author proposed is Morphological Reconstruction Based Segmentation, used to segment the solid cum cystic tumor of brain and is suggested after testing and observing various available methods and algorithms. The proposed method shows more precision amongst others and the processing time is also fast.
\end{abstract}

Index Terms-Image processin, medical diagnosis, object detection, tumors.

\section{INTRODUCTION}

Brain is a very complex structure and a vital organ and whole of the body functions depend upon its performance and activity. It is therefore, any kind of disorder and malfunctioning in the brain must be detected as quickly as possible in order to keep its operations intact. Numerous imaging modalities are available for anatomical investigations. MRI is an extremely valuable imaging technique for the anatomical investigations of diverse functions. Different MRI techniques are available to be used for dedicated purpose. Moreover, the manual calculations using the conventional techniques are often time consuming. At times the results are not precise and reliable, which may lead to missed tumor detection.

There is a need of semi or fully-automated system for the analysis of MRI images to save the radiologist's time. The system should be able to aid surgeons and other medical professionals, by providing an automated framework which is able to analyze the image data efficiently and above all make the whole process cost effective. Therefore, the main task is to investigate the limitations of existing facilities and to propose an automated system through searching, testing and applying proper procedures and algorithms in order to improve its quality. It would help replace the existing scenario with an imaging modality in which the machine can be programmed to detect tumors and resets its focus to reacquire data on the spot. This could improve diagnosis and reduce the time and cost involved in trials of new treatments. The time required to analyze each MRI image would also reduce considerably.

Manuscript received September 8, 2013; revised October 25, 2013.

Mashal Tariq and Attaullah Khawajah are with the N.E.D. University of Engineering \& Technology, Electronics Department, Karachi, Pakistan (email:mashalzafar79@gmail.com; atta@neduet.edu.pk).

Munawer Hussain is with the Department of Radiology, Dow University of Health \& Sciences, Ojha Campus, Karachi, Pakistan (e-mail: munawer66@yahoo.com).
This paper focuses on the research conducted for the automated detection of solid cum cystic tumor in brain using the T2 weighted image and contrast enhanced T1weighted image by morphological reconstruction. The primary objective of research is to present the solid cum cystic tumor in the form which is easily recognizable even by a nonprofessional.

This paper is divided into 4 sections: 1) describes methodology of proposed algorithm, 2) explains theoretical background of method, 3) shows experimental results and statistical parameters, and 4) enlightens conclusion and scope of future work.

\section{Methodology Adopted}

\section{A. Review Stage}

Segmentation is the most important and complicated stage of Digital Image Processing. In the case of brain tumor, segmenting a tumor out of brain is not an easy task. There are dynamic ranges of work in this discipline [1]-[6]. Going through available literature, it is observed that a lot of work is done on automation, to detect Soft Boundaries / Homogenous and Lesion Detection. Matei (2005) used Region Growing Threshold but it can only trace out the active part of tumor with one seed in the region of interest. In case of presence of Necrotic cells in tumor, it is needed to input more seeds to trace out the whole region of interest, requiring more human involvement and also the computations are complicated. Phooi (2005) treated T2 Weighted Image for detection of malignant lesion using three values i.e. shape, texture and contents. Moon (2002) worked on soft boundary tumors, proposed an extension to existing Expectation Maximization Segmentation. Murugavalli (2007) recommended the Neuro-Fuzzy Logic for the tumor segmentation. Macenko (2006) employed Morphological Watershed Segmentation for the tumor detection. Corso (2008) proposed Integrated Bayesian Model Classification on heterogeneous data. However, it is interesting to find out that no work is carried out for the Solid cum Cystic type of brain. The segmentation of tumor will be tested by applying algorithms to images.

\section{B. Initial Steps}

The segmentation of tumor will be tested by applying algorithms to images. Global Thresholding Method, Nearest Neighbor Operations, Region Growing Segmentation [7] and Watershed Segmentation were tested but led to failure. Reason being that on the T2 Image the cyst appears white where as on T1as dark. All the techniques work well for cystic part specifically for T2. For the solid part none of the techniques delivered required result. On the binary image other parts of the brain appear with the solid which is not the 
goal. Also contrast enhanced T1 Weighted Images are also tested which show promising result but only for the solid part. Reason being that contrast agent makes the solid appear brighter. Fig. 1. represents original MRI brain images of case $A$ in axial plane.
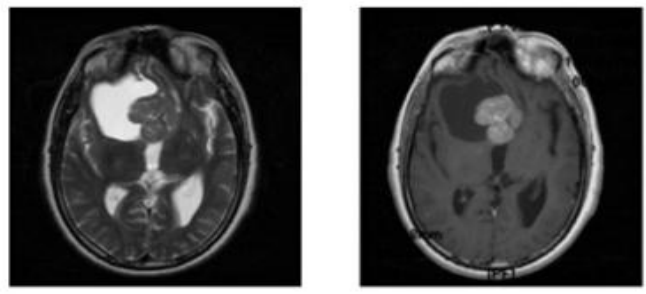

Fig. 1. Original images of case A: (a) T2 weighted (b) T1 weighted contrast enhanced.

\section{Methods and Materials}

The required dataset is collected from the Dow University of Health Sciences (Ojha Campus) from GE 1.5 Tesla MRI with the DICOM software. Specifically 4 brain tumor cases are arranged with their radiology reports alongside different slice thickness, in three planes i.e. axial, coronal, and sagittal with different techniques. For the paper, T2 Weighted and contrast enhanced T1 Weighted Images are selected, specifically their axial view. The platform used for the analysis is MATLAB 7. 7. 0. The proposed algorithm is tested on Microsoft windows XP (Professional), version 2002 with $2 \mathrm{~GB}$ of RAM. Intel Core 2 Duo, $1.83 \mathrm{GHz}$ processor type.

As the preliminary step all the images are converted to JPEG format with $100 \%$ quality. Size of T2 image of case $A$ is $512 \times 512$ pixels and where as size of contrast enhanced T1 image is $256 \times 256$ pixels. There is a need to resize the images of 256 pixels into 512 pixels to make them easy to compare. It is done with bi-cubic interpolation. All images of other cases are equal in size i.e. $512 \times 512$ pixels.

In this paper, we propose a very simple MRI image based technique to segment out Solid cum Cystic tumor. Various morphological operations are tested but morphological reconstruction [8] shows accurate results. Solid part is segmented using contrast enhanced $\mathrm{T} 1$ where as cystic part is taken from T2. The block diagram representation of our proposed algorithm is shown in Fig. 2.

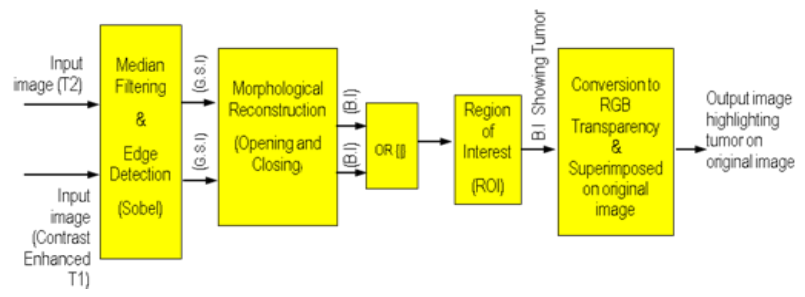

Fig. 2. Block diagram representation of proposed methodology.

\section{Theoretical Background of Methodology ADOPTED}

\section{A. Noise Reduction Filter}

The noise reduction filter is very important for the image enhancement. Here, the Median Filter is used where the median of the neighborhood pixels are used to find the output instead of mean. In Matlab it is implemented with medfilt 2 command. By default it uses 3-by-3 neighborhood.

\section{B. Sobel Edge Detector}

Edge detection is used to extract the information about the boundaries of objects in an image. For the particular purpose Sobel masks are used to detect the edges in the input image. Each image is treated with the convolution of two kernels. The following kernels in (1) are used to detect the horizontal $\left(h_{y}\right)$ and vertical $\left(h_{x}\right)$ edges.

$$
h_{x}=\left(\begin{array}{ccc}
1 & 0 & -1 \\
2 & 0 & -2 \\
1 & 0 & -1
\end{array}\right), h_{y}=\left(\begin{array}{ccc}
1 & 2 & 1 \\
0 & 0 & 0 \\
-1 & -2 & -1
\end{array}\right)
$$

Image output $(I(x, y))$ with edges can be obtained with the gradient magnitude. It can be calculated with (2) by taking the magnitude of the horizontal $\left(I_{Y}\right)$ and vertical gradient $\left(I_{X}\right)$ of each pixel.

$$
I(x, y)=\left[I x^{2}+I y^{2}\right]^{\frac{1}{2}}
$$

\section{Morphological Operations}

The morphological operations purely deal with shapes for Digital Image Processing. It is essential for image segmentation, object recognition and as a pre-processing steps [9]-[11]. It works with two sets of images, one is the original image and the other is the structuring element called the Kernels and Masks. The two basic morphological operations are Dilation and Erosion.

\section{1) Dilation and erosion}

Dilation thickens the object in an image by adding pixels to the boundaries of image. Its operation is highly dependent upon the shape and size of structuring element. In the set theory its operation can be defined as in (3). Equations (3) and (4) are based on [12].

$$
(f \oplus b)(x, y)=\max \left\{f\left(x-x^{\prime}, y-y^{\prime}\right) \mid\left(x^{\prime}, y^{\prime}\right) \epsilon D_{b}\right\}
$$

whereas $f$ is the input image, $b$ is the structuring element, $D_{b}$ is the domain of $b$ and $f(x, y)$ is assumed to equal $-\infty$ outside the domain of $f$. The structuring element is normally a flat pattern. The disk shape structuring element is used in research.

Erosion makes object thin and results in reducing object. Its operation can be defined mathematically as in (4). The notations have already described above.

$$
(f \Theta b)(x, y)=\max \left\{f\left(x-x^{\prime}, y-y^{\prime}\right) \mid\left(x^{\prime}, y^{\prime}\right) \epsilon D_{b}\right\}
$$

\section{2) Opening and closing}

These are other important morphological operations. In opening, erosion is followed by dilation of image. The morphological closing is dilation followed by erosion.

\section{Morphological Reconstruction}

It is a type of morphological transformation. The operation deals with two images and a structuring element. Whereas, in other operation; only input image and structuring element is required. In Matlab, it is implemented with (5). 
Out $=$ imreconstruct $($ marker, mask $)$

(5)
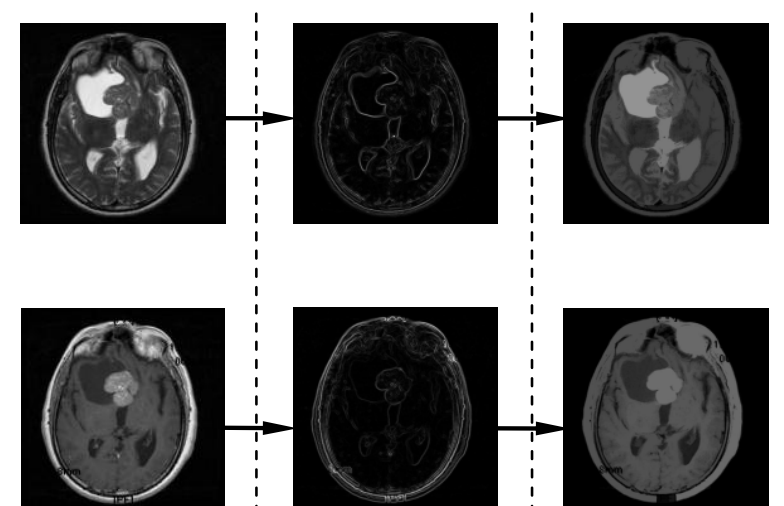

(a)

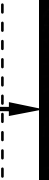

i

b)

(b)

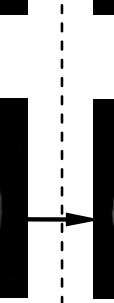

i

Fig. 3A. Resultant images of proposed methodology column (a) Median filter (b) Sobel edge detection (c) Morphological reconstruction.

The imreconstruct function uses the algorithm given in Vincent [13]. Opening by reconstruction shows better result than ordinary opening. The remaining object is exactly restored in this operation after erosion. The imreconstruct is used after imerode function for opening by reconstruction operation. It can be implemented in the following manner:

1) Dilating the opening by reconstructed image.

2) Taking the complements of dilated and eroded image.

3) Using complemented images in imreconstruct function.

4) The output of the previous step is complemented, which is the required output.

\section{E. OR Gate and Region of Interest}

The OR gate is used to combine both the solid and cystic part. Following it, imfill command of Matlab is used to fills holes in the binary image. As cystic portion is always with the solid so it fills holes around edges of image to merge them. Region of Interest is used to select tumor. As other parts of brain appear with tumor, imfill command makes the tumor appear larger so the larger area is selected as region of interest.

\section{F. Conversion to RGB Image and Transparency}

In the end, binary image is converted to RGB form by command label2rgb. Alphadata makes the image a transparency. Finally the transparency is superimposed on the original image highlighting the tumor.

\section{EXPERIMENTAL RESUlts}

In this Section, we present results of algorithm on all 4 cases. For the detection of different sizes of tumors in different regions, algorithm runs with different sizes of structuring elements. Results of case A are shown in detail, in Fig. 3A and 3B. It reveals images at each step of proposed method. Images in each column represent steps of algorithm. As it can be seen that first column represents imagses after median filtering and so on. It is shown that the solid part comes from the T1 weighted contrast enhanced and cyst part appears from T2 weighted image. OR logic is used to add both parts of tumors. And region of interest is used to select tumor. Finally tumor is highlighted in red on the original image. The results of other cases are summarized in Fig. 4. All the results clearly show the accuracy of algorithm.

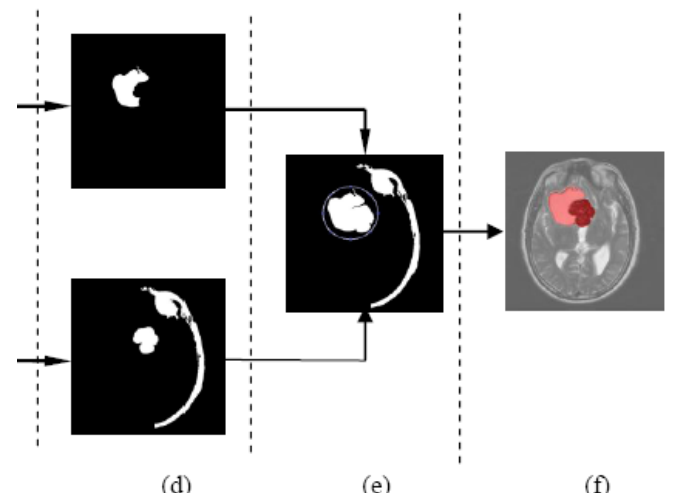

(d)

(e)

(f)

Fig. 3B. Resultant images of proposed methodology Column (d) Detected tumors (e) OR operation (f) Resultant image.
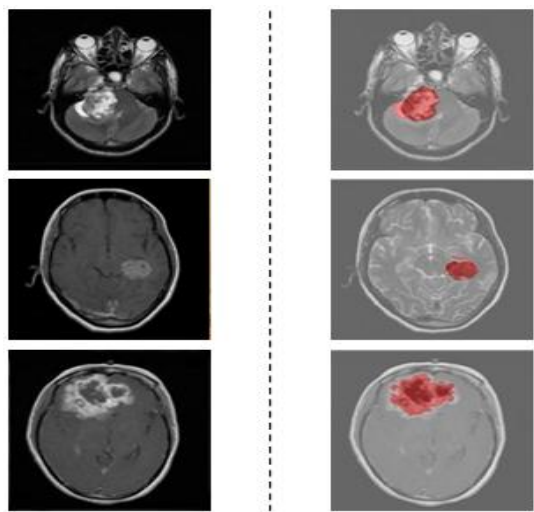

(a)

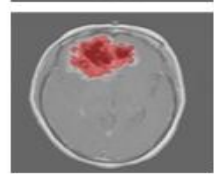

(b)

Fig. 4. Segmentation results of data set (a) Original images (b) Detected tumor of case B - D.

Table I shows some useful parameters. Total number of slices in each case and selected slice to work with is given. Area and execution time of software are also mentioned. Area is found out using the regionprop command of Matlab on binary image.

TABLE I: AREA AND EXECUTION TIME
\begin{tabular}{|c|c|c|c|c|}
\hline \multirow{2}{*}{ Cases } & Total Slices & Working Slice & Area (pixel) & Time (sec) \\
\hline A & 19 & 10 & 13751 & 18 \\
\hline B & 23 & 10 & 7050 & 12 \\
\hline C & 19 & 8 & 3763 & 25 \\
\hline D & 19 & 9 & 12427 & 22 \\
\hline
\end{tabular}

TABLE II: ERROR AND ACCURACY

\begin{tabular}{|c|c|c|}
\hline Cases & Error & Accuracy \\
\hline A & 0.999 & 99.001 \\
\hline B & 0.0222 & 99.778 \\
\hline C & 1.9998 & 98.0002 \\
\hline D & 0.9962 & 99.0038 \\
\hline
\end{tabular}

Table II shows the error and accuracy of tumor detection of all case. It is found out using imdistline function in Matlab, manually. Firstly, the dimension (dim1) of tumor on original image is noted and then segmented binary image of tumor is measured (dim2). Error is calculated with (6) and accuracy as in (7).

Error $=\operatorname{dim} 1-\operatorname{dim} 2$

$$
\text { Accuracy }=100 \text { - Error }
$$




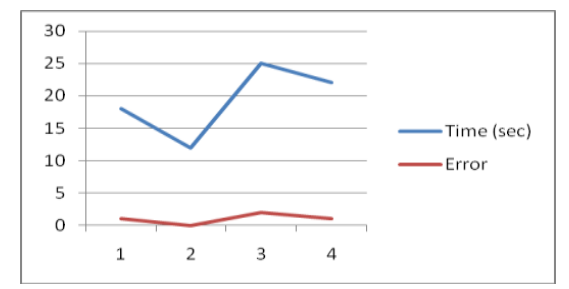

Fig. 5. Graphical representation of time and error.

Fig. 5 shows graphical representation of time and error with respect to individual case.

\section{CONCLUSIONS AND FUTURE WORKS}

The segmentation and recognition of object is an important step in digital image processing. Its application in bio-medical engineering has grown with time. It helps medical professionals in various ways. The proposed method will be helpful in making the detection of tumor by an automated process with accuracy.

The MRI imaging protocols are studied with the brain anatomy. Many segmentation techniques are tested and results are observed. All procedures after segmentation show other parts of brain with the tumor (not showing accuracy).

The MRI imaging protocols are studied with the brain anatomy. Many segmentation techniques are tested and results are observed. All procedures after segmentation show other parts of brain with the tumor (not showing accuracy).

The morphological operations show the desired result. Opening and Closing by morphological reconstruction shows better result than using only the Opening and Closing Morphological Operations. In this dissertation, solid cum cystic tumor is segmented out successfully from the image through Morphological Reconstruction. Results of dataset show accuracy with high precision. The tumor is highlighted in color on the original image showing precision of the projected procedure. On the average, the execution time is 19.25 seconds The proposed algorithm could save hours of radiologists' work. The precision of proposed method is highly reliable. It is as accurate as $98.946 \%$. Macenko (2006) recieved $90 \%$ accuracy in brain lesion detection where as Corso (2008) achieved 70\%. This clearly states the efficiency of proposed methodology.

The scope of future work includes, as proposed method uses single slice of T2 and contrast enhanced T1. It can be tested on other slices. The scope of future project encompasses segmenting the whole tumor by using a single technique of MRI. It can be done through in depth study of texture and intensities variations. The main objective is to design such a system that could be able to segment out all kind of brain tumor with accuracy and fast processing time.

This work can be extended to the $3 \mathrm{D}$ visualization of tumor, with its volume representation. It can be conducted with the softwares $3 D$ Slicer and the SPM. The later is supported by Matlab.

\section{ACKNOWLEDGMENT}

Mashal Tariq would like to thank Dr. Saad Qazi, Dr. Nazeer Khan and Dr. Raffique Khanani for their immense support and help.

\section{REFERENCES}

[1] P. Y. Lau, F. C. T. Voon, and S. Ozawa, "The detection and visualization of brain tumors on T2-weighted MRI images using multiparameter feature blocks," in Proc. IEEE Eng. in Medicine and Biology 27th Annu. Conf., Shanghai, 2005, pp. 5104-5107.

[2] M. Mancas, B. Gosselin, and B. Macq, "Segmentation using a region growing thresholding," in Proc. SPIE Image Process: Algorithms and Syst., San Jose, CA, 2005, vol. 5672, pp. 388-398.

[3] N. Moon, E. Bullitt, K. V. Leemput, and G. Gerig, "Automatic brain and tumor segmentation," in 2002 MICCAI, LNCS, vol. 2489, pp. 372-379.

[4] S. Murugavalli and V. Rajamani. (2007). An improved implementation of brain tumor detection using segmentation based on Neuro Fuzzy Technique. J. Comput.Sci. [Online]. 3(11). pp. 841-846. Available: http://www.scipub.org/fulltext/jcs/jcs311841-846.pdf

[5] M. Macenko, M. Celenk, and L. M. Ma, "Lesion detection using morphological watershed segmentation and modelbased inverse filtering," in ICPR, Hong Kong, vol. 4, pp. 679-682, 2006.

[6] J. J. Corso, E. Sharon, S. Dube, S. El-Saden, U. Sinha, and A. Yuille, "Efficient multilevel brain tumor segmentation with integrated Bayesian Model Classification Inverse Filtering," IEEE Trans. Med. Imag., vol. 27, pp. 629-640, 2008.

[7] Region-Growing. [Online]. Available: http://www.mathworks.com/matlabcentral/fileexchange/19084region-growing

[8] Morphological operations, Image Processing Toolbox User's Guide, Aksmlore, April 07, 2013

[9] W. A. Ulbeh, A. Moustafa, and Z. A. Alqadi. (2009). Gray image reconstruction. European J. Scientific Research. [Online]. 27. pp. 167-173, Available: http://www.eurojournals.com/ejsr_27_2_01.pdf

[10] C. Santhaiah, G. Anjan Babu, and M. Usha Rani. (July 2009). Graylevel morphological operations for image segmentation and tracking edges on medical applications. IJCSNS. [Online]. 9. pp. 131-136. Available: http://paper.ijcsns.org/07_book/200907/20090719.pdf

[11] R. Jones and I. Svalbe, "Algorithms for the decomposition of GrayScale Morphological Operation," IEEE Trans. Pattern Anal. Mach. Intell., vol. 16, pp. $581-588,1994$.

[12] R. C. Gonzalez, R. E. Woods, "Morphological image processing," Digital Image Processing Using Matlab, Fifth Impression, Delhi, India: Pearson, 2009,ch. 9, sec. 9.6, pp. 380-382

[13] L. Vincent, "Morphological grayscale reconstruction in image analysis: Applications and efficient algorithms," IEEE Trans. Image Process., vol. 2, pp. 176-201, 1993.

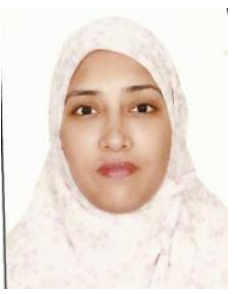

Mashal Tariq was born on October 24, 1979 , Karachi, Pakistan. She is a lecturer in College of Business Administration, Jeddah, Saudia Arabia. She received her B.E. in Electrical Engineering from NED University in 2002 and masters in Telecommunications from the NED in 2010 respectively. Her research interests include image processing, neural networking, integrating and simulation of data in $2 \mathrm{D}$ and $3 \mathrm{D}$.

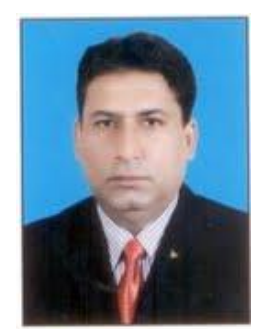

Attaullah Khawaja is a director, co-chairman and M.Engineering co-coordinator in NED University of Engineering and Technology, Karachi. He was born in 1970. He received his Ph.D. in Electronics in 2007 from China and M.Engineering in 2002 from NEDUET. His research interests include Neural network, Humanoid robotics and image processing using FPGA including tumor detection. He has above 20 publications. He has membership of PEC, IEEE, IACSIT and IAE.

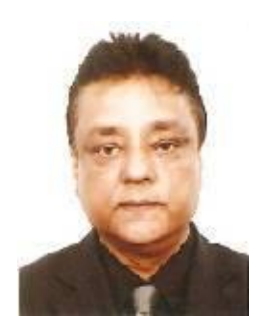

Munawar Hussain was born in 1957. He has MBBS, MCPS and FCPS in Diagnostic radiology. $\mathrm{He}$ is an assistant professor and project director, consultant radiologist in DOW Institute Radiology, Karachi. His research work includes Color Doppler Sonography in Erectile Dysfunction and Positioning retake causes in Digital Radiography. He has several publications in his name. 